Revista de

Contabilidade e

Organizações

www.rco.usp.br
DOI: http://dx.doi.org/10.11606/issn.1982-6486.rco.2020.172020
Journal of

Accounting and

Organizations

\title{
A ansiedade dos mestrandos e doutorandos em contabilidade
}

\author{
Anxiety in accounting graduate
}

Weverton Eugênio Coelho ${ }^{a}$, Eduardo Mendes Nascimento ${ }^{a}$

${ }^{a}$ Universidade Federal de Minas Gerais

Palavras-chave

Ansiedade.

Mestrado.

Doutorado.

Autoeficácia.

Motivação.
Keywords

Anxiety.

Master.

Doctorate.

Self-efficacy.

Motivation.

\section{Resumo}

Esta pesquisa teve como objetivo analisar o efeito que a motivação, a autoeficácia e variáveis sociodemográficas exercem sobre a ansiedade dos mestrandos e doutorandos em Contabilidade. A amostra foi composta por 246 mestrandos e 76 doutorandos de todos os programas de mestrado e doutorado em Contabilidade do Brasil. Os instrumentos de coleta de dados utilizados foram o Inventário de Ansiedade Traço, para determinar o nível de ansiedade; a Escala de Autoeficácia Geral Percebida, para levantar o nível de autoeficácia; e a Escala de Motivação Acadêmica, para determinar o nível de motivação intrínseca e extrínseca e de desmotivação. Os dados foram analisados através de regressão linear, utilizando a técnica Stepwise Backward. Os resultados revelaram uma predominância de ansiedade nas discentes do gênero feminino em comparação aos discentes do gênero masculino. Em relação ao objetivo primário do trabalho, concluiu-se que a ansiedade é negativa e significativamente impactada pela autoeficácia. Contudo, ela é positiva e significativamente impactada pela motivação extrínseca por controle externo e pela desmotivação. Concluiu-se, também, que preditores, como o nível de motivação para prosseguir nos estudos, ter idade abaixo de 24 anos, o tipo de pós-graduação (se mestrado ou doutorado), receber apoio psiquiátrico/psicológico, receber bolsa acadêmica, o desempenho e o gênero, são significativos para a análise da ansiedade.

\begin{abstract}
This research analyzes the effect of motivation, self-efficacy, and sociodemographic variables on the anxiety of master's and doctoral's accounting students. The sample consisted of 246 master's students and 76 doctoral students from all graduate accounting programs in Brazil. The study used the State-Trait Anxiety Inventory to collect data and determine the participants'level of anxiety; the General Self-Efficacy Scale to find their level of self-efficacy; and the Academic Motivation Scale to assess their level of intrinsic, extrinsic, and amotivation. Data analysis used backward stepwise linear regression. The results revealed a predominance of anxiety in female students and concluded that anxiety is negative and significantly affected by selfefficacy. It is also positive and significantly affected by extrinsic motivation by external control and by amotivation. Finally, the research identified significant predictors to analyze anxiety such as the level of motivation to continue studying, being under 24 years old, the type of graduate (whether master's or doctoral), receiving psychiatricl psychological support, receiving an academic scholarship, performance, and gender.
\end{abstract}

\section{Implicações Práticas}

Quanto mais amplo for o conhecimento sobre a situação do estudante, mais ampla é a possibilidade de implementação de políticas efetivas de intervenção, que visem atuar no sentido contrário a interações prejudiciais (caso existam). Assim, a busca por moduladores da ansiedade na pós-graduação está associada à procura de estratégias, que evitem ou diminuam o padecimento mental dos estudantes, a evasão ou sua formação insuficiente.

Copyright (C) 2020 FEA-RP/USP. Todos os direitos reservados. 


\section{INTRODUÇÃO}

A Contabilidade destaca-se como uma área importante do conhecimento científico, visto ser ela um ramo do conhecimento que provê agentes decisórios, capazes de atuar, de forma significativa, nas diversas vertentes que demandam o conhecimento contábil, ou seja, no controle econômico e patrimonial das empresas e demais entidades, na apuração de haveres e na avaliação de direitos e obrigações (Conselho Federal de Contabilidade, 2003). Para Iudícibus, Martins e Carvalho (2005), a contabilidade é um campo de conhecimento fundamental para a sociedade, por resultar de uma metodologia cuja racionalidade científica permite a captação e o processamento dos fatos econômicos e financeiros, que fornece informações para a tomada de decisões.

Esses agentes tomadores de decisões contábeis aprendem, desenvolvem e aperfeiçoam a prática contábil nos cursos de graduação e pós-graduação existentes. Quanto a isso, ainda, destaca-se o mestrado e no doutorado como espaço onde ocorre parte da formação de professores que atuarão na formação direta desses profissionais contábeis, visto a obrigatoriedade de que, no mínimo, um terço do corpo docente dos cursos de graduação seja composto de mestres e doutores ( Lei n. 9.394, 1996). Daí a importância educacional do ensino de Contabilidade, especialmente o stricto sensu (mestrado e doutorado), que, além de atuarem na produção científica (Conselho de Educação Superior, 1965), atuam na formação de futuros educadores, ou seja, professores responsáveis pela formação de futuros contadores.

Nesse sentido, Evans, Bira, Gastelum, Weiss e Vanderford (2018) destacam que, para que o indivíduo alcance todo o seu potencial e seus conhecimentos beneficiem todos os stakeholders (família, sociedade, programa de pós-graduação, por exemplo), é necessário que sua formação educacional seja saudável e motivadora. Podese afirmar, ainda, que há uma crescente preocupação quanto ao impacto das atuais condições do ensino na saúde mental dos estudantes, especialmente nos níveis educacionais de maior demanda, ou seja, as pós-graduações stricto sensu (Levecque, Anseel, Beuckelaer, Van der Heyden, \& Gisle, 2017).

Parte da preocupação com as condições do ensino de Contabilidade advém dos dados de pesquisas que comprovaram que os indivíduos que apresentam transtornos psicológicos tendem a apresentar um menor desempenho acadêmico (Reis, Miranda, \& Freitas, 2017). Nessa perspectiva, surge um debate importante sobre como a formação do profissional de contabilidade está ocorrendo, discussão que se busca apresentar neste artigo.

Pesquisas sobre o impacto do ambiente na pós-graduação estão, muitas vezes, ligadas a levantamentos que abrangem diversos cursos (Betz, 1978; Evans et al., 2018). Isso acontece porque, embora os transtornos ansiosos sejam quadros clínicos, os sintomas são primários e não derivados de outras condições psiquiátricas; os "sintomas ansiosos (e não os transtornos propriamente) são frequentes em outros transtornos psiquiátricos" (Castillo, Recondo, Asbahr, \& Manfro, 2000, p. 20). Assim, ao se trabalhar a ansiedade, a probabilidade de se captar a ocorrência de sintomas ansiosos derivados de outras condições psiquiátricas (como depressão, por exemplo) será mais abrangente no levantamento do indício de saúde mental do estudante de contabilidade.

Além de pesquisar o estado de ansiedade dos mestrandos e doutorandos e de buscar uma forma de se encontrar estratégias de intervenção, Weiner (1990) recomendou que o impacto de qualquer variável no processo educacional seja estudado, misturando-se variáveis que analisem emoções e perturbações. Nesse sentido, acreditase que tanto a motivação, conforme o modelo hierárquico de Vallerand (1997), em que a motivação é apresentada em dimensões e tipos (motivação intrínseca, motivação extrínseca e desmotivação); quanto a autoeficácia, conforme a proposição teórica de Bandura (1977), que propõe que a crença na autoeficácia pessoal é um importante fator cognitivo, motivacional e comportamental, são moduladores eficazes da ansiedade. Assim, a autoeficácia, por influenciar no empenho, na perseverança e na escolha da tarefa (Schunk, 1991); e a motivação intrínseca, por favorecer o aprendizado de alta qualidade (Ryan \& Deci, 2000), contribuem para um ambiente favorável ao desenvolvimento pleno dos estudantes, colaborando na diminuição da ansiedade.

Dessa forma, embora diversas pesquisas demonstrem a situação de saúde mental dos estudantes, observase uma lacuna em relação a como esse efeito ocorre, especificamente, no mestrado e doutorado em contabilidade e sobre qual o papel outras variáveis, como gênero, autoeficácia, motivação, desempenho, por exemplo, exercem nessa relação. Assim, a fim de compreender a situação dos mestrandos e doutorandos em contabilidade, abordando a referida lacuna, apresenta-se a proposta de responder à questão: Quais efeitos a motivação, a autoeficácia e variáveis sociodemográficas exercem sobre a ansiedade dos mestrandos e doutorandos em Contabilidade? Dessa maneira, neste trabalho, propõe-se, como objetivo, analisar o efeito que a motivação, a autoeficácia e variáveis sociodemográficas exercem sobre a ansiedade dos mestrandos e doutorandos em Contabilidade. 
O presente trabalho foi estruturado em cinco partes como elementos textuais, além de elementos prétextuais (resumo e palavras-chaves) e pós-textuais (referências). A parte textual está composta por esta que ora se encerra, na qual se apresenta a contextualização, o problema de pesquisa e os objetivos; a segunda é dedicada à revisão da literatura; na terceira parte faz-se a apresentação da metodologia do trabalho; na quarta parte são apresentados os dados e sua análise; e, por fim, tem-se a conclusão, exposta na quinta parte.

\section{REFERENCIAL TEÓRICO}

\subsection{Sofrimento mental na pós-graduação}

A pós-graduação é um marco na vida dos estudantespor significar muitas mudanças, que os impelem a se adaptarem a novas exigências, em muitos casos, são situações nunca antes vivenciadas (Tyssen \& Vaglum, 2002; Levecque et al., 2017). Esse contexto, sem dúvida, afeta não só a relação dos pós-graduandos com o meio acadêmico, mas também as suas relações familiares e o seu bem-estar físico e mental, ou seja, a pós-graduação introduz desafios demasiadamente complexos para a capacidade psíquica dos estudantes (Tyssen \& Vaglum, 2002; Levecque et al., 2017).

Quando o indivíduo não tem habilidades de enfrentamento, sejam elas baseadas nas suas experiências anteriores, sejam calcadas na sua capacidade de adaptação para lidar com aquelas demandas, ele pode ser vítima de sofrimento psíquico, entre os quais se destacam a ansiedade, a depressão, o estresse e outros distúrbios psicossomáticos e danos à saúde psicofisiológica (Horta, Horta, Horta, 2012; Andrade et al., 2016; Oliveira, 2019). Isso quer dizer que o sofrimento psíquico pode resultar em distúrbios psiquiátricos menores ou mesmo desencadear a instalação de episódios críticos de transtornos mentais graves ou persistentes (Horta et al., 2012).

Esse mal-estar psíquico é uma situação especialmente significativa nas instituições de ensino superior, considerando as suas taxas de prevalência, sendo esperado encontrar taxas entre $8 \%$ a $23 \%$ na sociedade em geral, mas, quando se isola o meio universitário, a prevalência de transtornos mentais pode chegar a $58 \%$ (Andrade et al., 2016; Oliveira, 2019). Essa é, certamente, uma indicação de que o ensino superior, em especial a pós-graduação, é uma ambiente de altas exigências psicoemocionais para os estudantes.

Essa situação se mostra mais preocupante considerando que problemas graves podem ser desencadeados por essa exaustão emocional dos estudantes. Para além dos transtornos envolvendo altos níveis de ansiedade, estresse e depressão, há repercurssões para os universitários em seu desempenho (Reis et al., 2017), além da possibilidade de uso abusivo de substâncias, como drogas (Horta et al., 2012; Oliveira, 2019), e, em casos extremos, de suicídio (Garcia-Williams, Moffitt \& Kaslow, 2014; Tyssen \& Vaglum, 2002).

\subsection{Ansiedade}

Dentre as diversas definições de ansiedade, destaca-se a de Freud, que a definiu como algo que se sente como fruto de um conflito mental, enfatizando que a ansiedade age na motivação comportamental e auxilia a lidar com situações ameaçadoras, mas também, quando apresentada de forma intensa, é um distúrbio psiquiátrico (Weiner \& Craighead, 2009). Dessa forma, observa-se que a ansiedade apresenta dois lados: de um lado ela se apresenta como passageira e seus efeitos são caracterizados como favoráveis ao comportamento; de outro, ela se apresenta como aspecto persistente do comportamento e é tratada como patológica, pelos efeitos desfavoráveis que desencadeia no organismo (Neiva, 2010).

Segundo Spielberger (1972), para o estudo da ansiedade, é necessário distingui-la como ansiedade estado e ansiedade traço, sendo que:

O estado de ansiedade (A-estado) é conceitualizado como um estado emocional transitório ou condição do organismo humano que é caracterizado por sentimentos desagradáveis de tensão e apreensão conscientemente percebidos, e por aumento na atividade do sistema nervoso autônomo. Escores em A-estado podem variar em intensidade e flutuar no tempo. O traço de ansiedade (A-traço) refere-se a diferenças individuais relativamente estáveis em propensão à ansiedade [...] (Biaggio, Natalício, \& Spielberger, 1977, pp. 31-32). 
Em outras palavras, o traço de ansiedade está relacionado à característica de 'ser' ansioso, enquanto o estado de ansiedade está relacionado ao foto de 'estar' ansioso (Spielberger, 1966). Assim, observa-se que o traço de ansiedade é um conceito mais estável ao longo do tempo, sendo menos volátil a condições ambientais momentâneas, por isso ele foi escolhido para ser tratado neste estudo. A Figura 1 apresenta a concepção do EstadoTraço de ansiedade e como ele atua no comportamento.

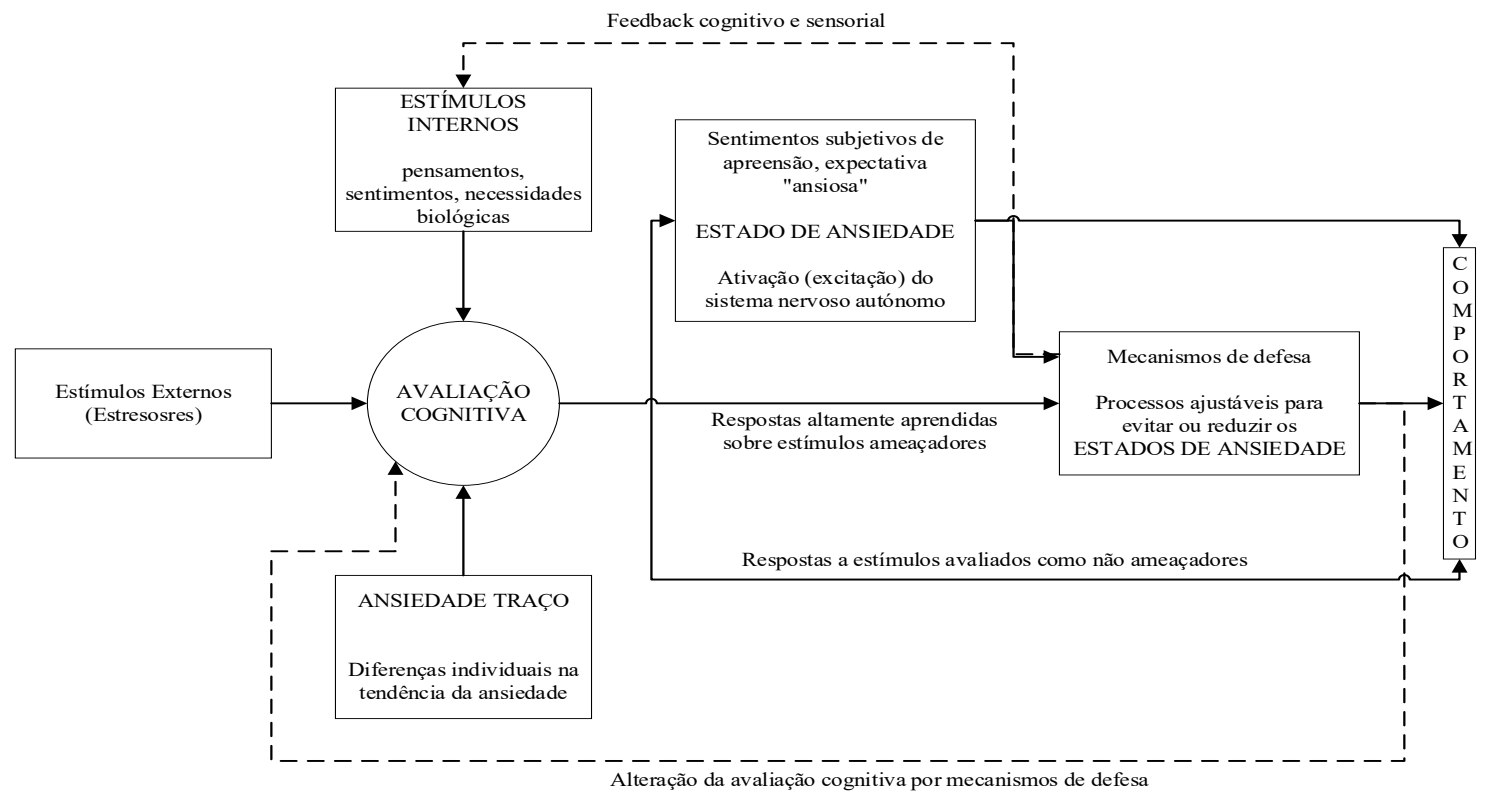

Figura 1. Concepção do Estado-Traço de Ansiedade de Spielberg (1966)

Fonte: Spielberg $(1966$, p. 17)

Conforme observa-se na Figura 1, a avaliação cognitiva de um estímulo externo causa a ativação do estado de ansiedade e ativa os mecanismos de defesa do organismo. Essa avaliação pode desencadear uma sequência de comportamentos, que fazem, por exemplo, com que o indivíduo evite a situação, ou use manobras defensivas (Spielberger, 1966). Trazendo essa informação para o contexto educacional, percebe-se que o comportamento de evitação é prejudicial ao desempenho, pois atua de forma contrária ao estabelecimento de metas, do mesmo modo que a ativação exagerada do sistema nervoso autônomo pode debilitar a aprendizagem.

\subsection{Autoeficácia}

A referência no estudo de autoeficácia é Bandura (1977), o qual teorizou um mecanismo cognitivo que explica e modula o comportamento em diversos domínios na execução de tarefas, definindo esse mecanismo como crença, percepção ou expectativa de autoeficácia. Schunk (1991) esclarece que, desde o artigo seminal de Bandura diversas pesquisas confirmaram a função da autoeficácia como um mecanismo capaz de realizar mudanças comportamentais e predizer resultados, como realizações acadêmicas e escolha de carreira.

No meio educacional, "Autoeficácia refere-se a crenças sobre as capacidades de uma pessoa para aprender ou executar comportamentos em níveis designados" (Schunk \& Pajares, 2001, p. 2), ou seja, é uma crença pertencente à classe de 'expectativas' relacionadas ao 'eu' quanto ao autojulgamento do indivíduo de ser capaz de realizar determinada tarefa educacional com um nível de qualidade exigida (Bzuneck, 2001).

A percepção de autoeficácia pressupõe que, para realizar uma atividade, o indivíduo possui as habilidades necessárias, as quais dizem respeito ao conjunto de ações que devem ser empregadas para se atingir determinado comportamento, por isso o indivíduo que se considera mais autoeficaz mobiliza recursos cognitivos mais amplos e, consequentemente, obtém um melhor desempenho (Nogueira \& Mesquita, 1992). 
Bandura (1977) também postulou que a percepção de autoeficácia pode ser criada, reforçada ou reduzida, já que sofre influência do meio através de fontes de informação, como: suas performances pessoais, sua experiência vicária, persuasão verbal de terceiros e através da interpretação do seu estado emocional. As realizações de desempenho são a fonte mais influente de autoeficácia e dizem respeito às realizações passadas do indivíduo, finalizadas com sucesso.

As experiências vicárias também são uma importante fonte de autoeficácia, referindo-se à expectativa derivada da observação. Assim, embora não envolvido diretamente na tarefa, o sucesso de outro indivíduo, que é considerado um semelhante, transmite a crença de que o próprio sujeito também é capaz de realizar aquele comportamento (Serpa, 2012). A persuasão verbal, terceira fonte de autoeficácia é o feedback, comunicado ao estudante, preferencialmente, sobre suas qualidades positivas.

Por fim, as pessoas julgam sua autoeficácia também por intermédio da interpretação do seu estado emocional. Bandura (1977) explica que, como a ativação emocional alta debilita o desempenho, o sucesso é maior naqueles indivíduos que não são acometidos por excitação aversiva, já que ela pode despertar níveis altos de ansiedade, que excedem o medo real experimentado. As informações derivadas das fontes de autoeficácia passam por um processamento cognitivo que levam em conta o nível, a generalidade e a força da crença de autoeficácia, além dos fatores contextuais e situacionais, podendo ou não serem integradas à crença de autoeficácia do indivíduo (Bandura, 1977).

\subsection{Motivação}

Além da diversidade de significados para o termo motivação, existem ainda diversas abordagens psicológicas (Witter, 1984), como, por exemplo, a abordagem psicanalítica, a qual interpreta a motivação como sendo direcionada pelo inconsciente com o objetivo de satisfazer aspirações do desejo e da sexualidade, e a Teoria de Maslow, pela qual a motivação é categorizada segundo uma hierarquia de importâncias (Bueno, 2002; Maslow, 1943).

Dentre as teorias motivacionais disponíveis, este estudo utilizou da Teoria da Autodeterminação (Self Determination Theory), elaborada por Deci e Rayan nos anos de 1970 (Araujo, 2015), influenciados pelas proposições de White (1975). Essa teoria foi, contemporaneamente, tratada pelos próprios autores (Ryan \& Deci, 2000), que confirmaram a proposição de um continuum de autodeterminação, e esclareceram que, a Teoria da Autodeterminação distingue os diferentes tipos de motivação, baseando-se nas diferentes razões ou objetivos que dão origem a uma ação, sendo que a distinção mais básica reside entre motivação intrínseca e motivação extrínseca. Isto é:

(1) motivação intrínseca - a motivação para buscar uma atividade simplesmente pelo prazer ou satisfação que é derivada dela; (2) motivação extrínseca - exercer uma atividade com base no senso de obrigação, ou como um meio para um fim, e (3) desmotivação - a ausência de intenção ou motivação para exercer uma atividade devido a não valorizar a atividade, sentirse incompetente ou incapaz obter um resultado desejado (Fairchild, Horst, Finney, \& Barron, 2005, p. 332).

Além de testada e confirmada (Fairchild et al., 2005) a teoria da autodeterminação, também se considerou uma macroteoria da motivação, reconhecida e referenciada internacionalmente no campo educacional (Araujo, 2015). Dessa forma, por possuir estas três dimensões de análise: motivação extrínseca (subescalas: introjeção, controle externo, identificação); motivação intrínseca (subescalas: para realização, para saber, e para vivenciar estímulos); e dimensão da desmotivação, a teoria da autodeterminação foi a escolhida para ser utilizada como base para as análises motivacionais deste estudo (Vallerand, 1997; Viana, 2012). 


\section{METODOLOGIA}

\subsection{Instrumentos de coleta de dados}

O instrumento de coleta de dados foi constituído de um bloco inicial, contendo as variáveis sociodemográficas, seguido pelo inventário de ansiedade traço, squenciado pela escala geral de autoeficácia e, por fim, pela escala de motivação acadêmica. O documento de registro e aprovação deste estudo pela Comissão Nacional de Ética em Pesquisa recebeu o n. 97431018.1.0000.5149. Tanto o instrumento inicial passou por prétestes, quanto as três escalas utilizadas, mesmo já contando com validação nacional. Durante a aplicação do instrumento, um TCLE (Termo de Consentimento Livre e Esclarecimento) foi apresentado aos participantes e garantido o anonimato a todos, já que nenhuma idenficiação lhes foi solicitada.

Para mensurar a ansiedade, foi utilizado o Inventário de Ansiedade Traço de Spielberger e colaboradores (Spielberger \& Gorsuch, 1966; Spielberger, Gorsuch, \& Lushene, 1970), composto por uma escala tipo Likert de 4 pontos, com o objetivo de determinar a ansiedade que o indivíduo sente na maior parte do tempo. Esse instrumento foi traduzido e validado por Biaggio et al. (1977).

A autoeficácia foi medida por meio da versão nacional de Teixeira, Dias e Dell’Aglio (2012), da escala desenvolvida por Schwarzer e Jerusalem, denominada de Escala de Autoeficácia Geral Percebida, a qual consiste em uma escala tipo Likert de 10 itens, cada um com 4 pontos (Schwarzer, Bäßler, Kwiatek, Schröder, \& Zhang, 1997).

Por fim, para medir a motivação, o instrumento utilizado foi a Escala de Motivação Acadêmica, desenvolvida por Vallerand et al., (1992), a partir da versão francesa Echelle de Motivation en Education (EME). Esse instrumento é composto por 28 itens, dispostos em uma escala tipo Likert de 7 pontos. Ressalta-se que a versão nacional utilizada da Escala de Motivação Acadêmica serviu de base ao estudo de Sobral (2003). Ademais, resta dizer que os resultados dos estudos para as três escalas apoiam a validade e confiabilidade dos instrumentos no ambiente universitário.

\subsection{Tratamento dos dados}

Para sistematização e análise dos dados, foram utilizados os softwares Microsoft Excel ${ }^{\circledR}$ e Stata $12 ®$, e as análises utilizaram um nível de significância mínimo de 10\%. As variáveis deste estudo foram escolhidas com base na plataforma teórica ou percepção do autor quanto à influência da variável na ansiedade. As variáveis utilizadas no modelo de regressão estão descritas no Quadro 1.

Utilizando essas variáveis, descritas, foi gerado um modelo de Regressão Linear múltipla através da técnica Stepwise Backward, em que a variável dependente foi a ansiedade. As variáveis de controle foram: idade, gênero, estado civil, tipo de pós-graduação, suporte, residência e demanda. As variáveis explicativas foram: desempenho, motivação para prosseguir, expectativa de resultado, autoeficácia, motivação intrínseca para saber, motivação intrínseca para realização, motivação intrínseca para vivenciar estímulos, motivação extrínseca por identificação, motivação extrínseca por introjeção, motivação extrínseca por controle externo, e desmotivação, conforme apresentado na equação 1 .

$$
\begin{aligned}
& \text { Ansiedade }=\alpha_{1}+\beta_{1} \text { Dummy }_{\text {Idade }}+\beta_{2} \text { Dummy }_{\text {Genero }}+\beta_{3} \text { Dummy }_{\text {Est Civil }}+\beta_{4} \text { Dummy }_{\text {Tipo Pos }}+\beta_{5} \text { Dummy }{ }_{\text {Suporte }}+ \\
& \beta_{6} \text { Dummy }_{\text {Residencia }}+\beta_{7} \text { Dummy } \text { Demanda }+\beta_{8} \text { Desempenho }+\beta_{9} \text { Motivação_Prosseguir }+\beta_{10} \text { Expectativa_Resultado } \\
& +\beta_{11} \text { Autoeficacia }+\beta_{12} M I \text { Saber }+\beta_{13} M I \text { Realização }+\beta_{14} M I \text { Estímulos }+\beta_{15} M E \_ \text {Identificação }+\beta_{10} M E_{-} \\
& \text {Introjeção }+\beta_{17} M E \_C o n t r o l e+\beta_{18} \text { Desmotivação }
\end{aligned}
$$




\begin{tabular}{|c|c|c|}
\hline Variável & Descrição & Mensuração \\
\hline Ansiedade & Descreve a ansiedade & Valor numérico variando de ' 20 ' a ' 80 ' \\
\hline Idade & Descreve a idade & $\begin{array}{l}\text { Variável dummy para faixa de idade a cada } \\
5 \text { anos }\end{array}$ \\
\hline Gênero & $\begin{array}{l}\text { Descreve o gênero com o qual o discente } \\
\text { se identifica }\end{array}$ & $\begin{array}{l}\text { Variável dummy de valor ' } 1 \text { ' se discente } \\
\text { for do gênero masculino e ' } 0 \text { ' se discente } \\
\text { for do gênero feminino }\end{array}$ \\
\hline Estado civil & Descreve o estado civil & $\begin{array}{l}\text { Variável dummy de valor ' } 1 \text { ' se discente } \\
\text { for casado / união estável e '0' nas demais } \\
\text { condições }\end{array}$ \\
\hline Tipo de pós-graduação & $\begin{array}{l}\text { Descreve o tipo de pós-graduação que o } \\
\text { discente está cursando }\end{array}$ & $\begin{array}{l}\text { Variável dummy para cada tipo de pós- } \\
\text { graduação }\end{array}$ \\
\hline Residência & $\begin{array}{l}\text { Descreve se o discente teve que se mudar } \\
\text { do local que morava para cursar a pós- } \\
\text { graduação, perdendo o suporte familiar }\end{array}$ & $\begin{array}{c}\text { Variável dummy para cada tipo de opção } \\
\text { de residência }\end{array}$ \\
\hline Desempenho & $\begin{array}{l}\text { Descreve a pontuação média que o } \\
\text { discente obteve na pós-graduação }\end{array}$ & Valor numérico de '0’ a ‘ 100 ' \\
\hline Suporte & $\begin{array}{l}\text { Descreve se o discente obteve suporte na } \\
\text { forma de bolsa de mestrado ou se solicitou } \\
\text { apoio psicológico }\end{array}$ & $\begin{array}{l}\text { Variável dummy para cada opção de } \\
\text { resposta }\end{array}$ \\
\hline Demanda & $\begin{array}{l}\text { Descreve se o discente trabalhou durante a } \\
\text { integralização dos créditos }\end{array}$ & $\begin{array}{l}\text { Variável dummy que assumiu o valor de: } \\
\text { '1' para "Sim" e '0' para "Não" }\end{array}$ \\
\hline Motivação para prosseguir & $\begin{array}{l}\text { Descreve a motivação do discente de } \\
\text { continuar estudando }\end{array}$ & Valor numérico variando de '0’ a ' 100 ' \\
\hline Expectativa de resultado & $\begin{array}{l}\text { Descreve a nota que o discente esperava } \\
\text { obter ao ingressar na pós-graduação }\end{array}$ & Valor numérico que varia de '0' a '100' \\
\hline Autoeficácia & Descreve a autoeficácia & Valor numérico que varia de ' 10 ' a 40 ' \\
\hline MI* para saber & $\begin{array}{c}\text { Descreve a motivação intrínseca para } \\
\text { saber }\end{array}$ & Valor numérico que varia de '4' a '28' \\
\hline MI para realização & $\begin{array}{c}\text { Descreve a motivação intrínseca para } \\
\text { realização }\end{array}$ & Valor numérico que varia de '4' a '28' \\
\hline MI estímulos & $\begin{array}{c}\text { Descreve a motivação intrínseca para } \\
\text { vivenciar estímulos }\end{array}$ & Valor numérico que varia de '4' a '28' \\
\hline ME** identificação & $\begin{array}{c}\text { Descreve a motivação extrínseca por } \\
\text { identificação }\end{array}$ & Valor numérico que varia de '4' a '28' \\
\hline ME introjeção & $\begin{array}{c}\text { Descreve a motivação extrínseca por } \\
\text { introjeção }\end{array}$ & Valor numérico que varia de '4' a '28' \\
\hline ME controle externo & $\begin{array}{l}\text { Descreve a motivação extrínseca por } \\
\text { controle externo }\end{array}$ & Valor numérico que varia de '4' a '28' \\
\hline Desmotivação & Descreve a desmotivação & Valor numérico que varia de '4' a '28' \\
\hline
\end{tabular}

Quadro 1. Variáveis utilizadas no estudo 


\subsection{Hipóteses de pesquisa}

Observam-se, segundo o modelo hierárquico de Vallerand (1997), consequências positivas e negativas ligadas às dimensões motivacionais, em que as motivações intrínsecas geram consequências mais positivas no comportamento, consequentemente reduzindo a ansiedade. Por outro lado, as dimensões da motivação extrínseca e a desmotivação geram consequências mais negativas, consequentemente aumentando a ansiedade (Vallerand, 1997).

Outrossim, quando se julga mais autoeficaz, o estudante reduz a ansiedade experimentada ao se confrontar com uma tarefa, conforme encontrado na pesquisa de Smith (1989). Desse modo, a autoeficácia deve ser negativa e significativamente relacionada à ansiedade. Por fim, a ansiedade tem demonstrado ser maior nos discentes do gênero feminino (Iqbal, Gupta, \& Venkatarao, 2018). Dessa maneira, e segundo a revisão de literatura apresentada, estabeleceram-se as seguintes hipóteses:

$\mathbf{H}_{1}$ : A autoeficácia impacta negativa e significativamente a ansiedade.

$\mathbf{H}_{2}$ : As dimensões da motivação extrínseca impactam positiva e significativamente a ansiedade.

$\mathbf{H}_{3}$ : A desmotivação impacta positiva e significativamente a ansiedade.

$\mathbf{H}_{4}$ : As dimensões da motivação intrínseca impactam negativa e significativamente a ansiedade.

$\mathbf{H}_{5}$ : As discentes do gênero feminino são estatística e significativamente mais ansiosas que os discentes do gênero masculino.

\section{APRESENTAÇÃO E DISCUSSÃO DOS RESULTADOS}

O envio online do instrumento resultou em uma amostra de 246 mestrandos e 76 doutorados de todos os programas de mestrado e doutorado em contabilidade do Brasil. Esses estudantes declararam ter 38 anos em média, ser a maioria do sexo feminino (52\%), ser casado (a) (48\%), que não buscaram apoio psicológico $(90 \%)$ nem bolsa (61\%), mas a maioria declarou trabalhar durante a obtenção de créditos $(65 \%)$, dados que podem ser constatados na Tabela 1.

Tabela 1. Estatística descritiva da amostra

\begin{tabular}{|c|c|c|c|}
\hline \multicolumn{2}{|c|}{ Variável } & \multirow{2}{*}{$\begin{array}{c}\text { Total } \\
169\end{array}$} & \multirow{2}{*}{$\begin{array}{c}\text { Relativo } \\
52 \%\end{array}$} \\
\hline \multirow{3}{*}{ Gênero } & Feminino & & \\
\hline & Masculino & 153 & $48 \%$ \\
\hline & Casodo(a) ou em união estável & 156 & $48 \%$ \\
\hline \multirow[t]{2}{*}{ Estado Civil } & Solteiro(a) & 150 & $47 \%$ \\
\hline & Outros & 16 & $5 \%$ \\
\hline \multirow{2}{*}{ Titulando em } & Mestrado & 246 & $76 \%$ \\
\hline & Doutorado & 76 & $24 \%$ \\
\hline \multirow{2}{*}{ Residência } & Mudou & 273 & $85 \%$ \\
\hline & Não mudou & 49 & $15 \%$ \\
\hline \multirow{2}{*}{ Apoio Psicológico } & Sim & 33 & $10 \%$ \\
\hline & Não & 289 & $90 \%$ \\
\hline \multirow{2}{*}{ Bolsa } & $\operatorname{Sim}$ & 126 & $39 \%$ \\
\hline & Não & 196 & $61 \%$ \\
\hline \multirow{2}{*}{ Demanda } & Trabalha & 209 & $65 \%$ \\
\hline & Não trabalha & 113 & $35 \%$ \\
\hline
\end{tabular}

Fonte: elaborada pelos autores

Esta pesquisa se propôs a testar se e como, nos estudantes stricto sensu, o comportamento da ansiedade varia, de acordo com o gênero. Cabe ressaltar que o Alfa de Cronbach das três escalas analisadas foram acima de 0,7, demostrando a confiabilidade dos instrumentos. Na Tabela 2, apresentam-se as médias do escore da escala de ansiedade geral e por gênero. 
Tabela 2. Estatísticas da Ansiedade Geral e por gênero

\begin{tabular}{lccc}
\hline \multicolumn{1}{c}{ Descrição } & Geral (N = 322) & Feminino (N = 169) & Masculino ( $=\mathbf{1 5 3})$ \\
\hline Média & 42,3 & 44,9 & 39,4 \\
Mediana & 41,0 & 44,0 & 38,0 \\
Desvio padrão & 11,4 & 11,6 & 10,4 \\
Mínimo & 20,0 & 20,0 & 22,0 \\
Máximo & 75,0 & 72,0 & 75,0 \\
$1^{\circ}$ Quartil & 33,0 & 37,0 & 32,0 \\
\hline
\end{tabular}

Fonte: elaborada pelos autores

O gênero feminino alcançou, em média, 42,3 pontos, e o gênero masculino, em média, 39,4 ponto. Os escores de ansiedade traço obtidos com a análise do gênero masculino e feminino foram maiores do que os escores do estudo de Spielberger, Gorsuch e Lushene (1979), que analisou uma amostra de estudantes brasileiros universitários do Rio de Janeiro (gênero feminino 41,3 pontos; gênero masculino 38,04 pontos).

Já o estudo de Borine (2011), ao pesquisar estudantes universitários de São Paulo e Roraima, encontrou que os discentes do gênero masculino tiveram o escore de 42,4 pontos e os discentes do gênero feminino 47,0 pontos, no questionário de ansiedade traço. Ou seja, esses resultados são superiores aos encontrados neste estudo. Por conta dessa variabilidade de resultados, ainda que a população seja de estudantes universitários, Spielberger et al. (1979) orientam que a análise da ansiedade seja feita considerando a população em que o questionário é aplicado.

Por essa razão, a distribuição da ansiedade neste estudo considerou tercis da população estudada. Ou seja, como o valor mínimo da escala foi 20 e o valor máximo foi 75, a amplitude da amostra é de 55 pontos, e a divisão desse valor por três nos informa a amplitude de cada tercil. Dessa maneira, o primeiro tercil varia de 20 pontos a 38,33 pontos; o segundo tercil, de 38,34 pontos a 56,67 pontos; e o terceiro tercil, de 56,68 pontos a 75 pontos, considerando-se o primeiro tercil de baixa ansiedade, o segundo de moderada ansiedade e o terceiro de alta ansiedade.

Observando-se a distribuição proposta constatou-se que, na amostra, 39,1\% (126 discentes) possuem ansiedade baixa, 48,1\% (155 discentes) possuem ansiedade moderada, e 12,7\% (41 discentes) possuem ansiedade alta. Dividindo-se esse resultado por gênero, observa-se que, dos $39,1 \%$ dos discentes que possuem ansiedade baixa, 38,9\% (49 discentes) são do gênero feminino e $61,1 \%$ são do gênero masculino ( 77 discentes); dos $48,1 \%$ dos discentes que possuem ansiedade moderada, $57,4 \%$ (89 discentes) são do gênero feminino e 42,6\% (66 discentes) são do gênero masculino; e, dos 12,7\% dos discentes que possuem ansiedade alta, 75,6\% (31 discentes) são do gênero feminino e 24,4\% (10 discentes) são do gênero masculino. Assim, constata-se que os mestrandos do gênero feminino estão mais presentes no segundo e, especialmente, no terceiro tercil da amostra em comparação aos discentes do gênero masculino.

Diante desse fato, entendeu-se necessário investigar se os valores médios de ansiedade do gênero feminino são estatisticamente maiores que os valores médios de ansiedade do gênero masculino. Assim, aplicou-se o teste Mann-Whitney de diferença de médias. $\mathrm{O}$ p-valor $=0,00$ demonstra que o gênero masculino apresenta escore de ansiedade significativa e estatisticamente menor que os escore do gênero feminino.

Os resultados apresentados estão conforme os encontrados na literatura, onde, sistematicamente, o gênero feminino apresenta um escore de ansiedade maior que o gênero masculino, ainda que o curso e o tipo de instituição sejam diferentes (Simon \& Thomas, 1983). Diante do exposto, a hipótese $\mathbf{H}_{5}$ : As discentes do gênero feminino são estatística e significativamente mais ansiosas que os discentes do gênero masculino, não foi rejeitada.

Em relação às demais variáveis da pesquisa, foi constatado que a média obtida, declarada pelos respondentes, foi de 85,0 pontos menor em apenas 0,8 pontos que a expectativa de resultado, o que não foi considerado estatisticamente diferente (teste de normalidade $-\mathrm{p}$-valor $<0,01$, teste de associação $-\mathrm{p}$-valor $<0,01$ ). A motivação para prosseguir alcançou aproximadamente $64 \%$ do escore máximo, enquanto a percepção de autoeficácia dos estudantes foi de $80 \%$ do escore máximo. Nesse sentido, chamou atenção que a Motivação Interna para o Saber ficou em 78\% da escala e Motivação Externa Identificação em 76\%. Esses resultados obtidos são apresentados na Tabela 3. 
Tabela 3. Estatística descritiva das variáveis

\begin{tabular}{lccccccc}
\hline \multicolumn{1}{c}{ Variável } & Média & Mediana & Moda & Mínimo & Máximo & Desvio padrão & Variância \\
\hline Idade & 33,7 & 31 & 27 & 18 & 68 & 9,2 & 84,5 \\
Desempenho & 85,0 & 85 & 85 & 65 & 100 & 7,3 & 53,0 \\
Expectativa de resultado & 85,8 & 90 & 90 & 0 & 100 & 10,7 & 113,5 \\
Motivação para prosseguir & 64,4 & 72 & 100 & 0 & 100 & 31,6 & 996,9 \\
Autoeficácia & 32,1 & 32 & 33 & 19 & 40 & 4,5 & 20,4 \\
MI* para saber & 22,0 & 23 & 28 & 4 & 28 & 4,9 & 24,5 \\
MI para realização & 20,5 & 21 & 28 & 4 & 28 & 5,9 & 35,0 \\
MI estímulos & 18,5 & 19 & 20 & 4 & 28 & 6,1 & 37,0 \\
ME** identificação & 21,3 & 22 & 28 & 4 & 28 & 5,2 & 27,4 \\
ME introjeção & 18,0 & 19 & 28 & 4 & 28 & 6,7 & 44,8 \\
ME controle externo & 20,3 & 22 & 28 & 4 & 28 & 6,6 & 44,0 \\
Desmotivação & 7,5 & 5 & 4 & 4 & 28 & 5,0 & 25,1 \\
\hline
\end{tabular}

Fonte: elaborada pelos autores

Nota: *MI=Motivação Intríseca/Interna **ME=Motivação Extrínseca/Externa.

\subsection{Regressão de Ansiedade}

Após a estimação do modelo, foram realizadas as validações requeridas para o método empregado, ou seja, multicolinearidade, normalidade de resíduos, correlação do erro com as variáveis, média do erro, forma funcional e heterocedasticidade, em que todos os resultados foram satisfatórios, com exceção da normalidade dos resíduos e da heterocedasticidade. Para o primeiro problema considerou-se o teorema do limite central (Wooldridge, 2010). Para a heterocedasticidade, o modelo Stepwise Backward foi reestimado, utilizando a correção dos erros robustos de White. O modelo final é apresentado na Tabela 4.

Tabela 4. Resultado do modelo de regressão Ansiedade stepwise backward com erros robustos de White

\begin{tabular}{lccc}
\hline \multicolumn{1}{c}{ Grupo } & Variável & Coeficiente & P-valor \\
\hline Constante & Constante & 67,83 & 0,00 \\
& D_Idade24 & 3,25 & 0,04 \\
Perfil do discente & D_GENERO & $-2,99$ & 0,00 \\
& D_Mestr_Doc2 & 2,27 & 0,03 \\
Desempenho & Pontuação_Obtida & 0,10 & 0,08 \\
Suporte & D_Apoio_Bolsa & 1,68 & 0,09 \\
Autoeficácia & D_Apoio_Psico & 7,70 & 0,00 \\
Motivação para prosseguir & Autoeficácia & $-1,25$ & 0,00 \\
Motivação & Motivação_Prosseguir & $-0,04$ & 0,02 \\
Desmotivação & ME_Controle & 0,23 & 0,00 \\
\hline
\end{tabular}

$\mathrm{R}^{2}$ ajustado $=59,19 \%$ Prob. $>\mathrm{F}=0,00$

Fonte: elaborada pelos autores

Com relação às variáveis estatisticamente significativas da regressão, constatou-se que a idade é um fator relevante ao se analisar a ansiedade, visto que, conforme apresentado na Tabela 4, os discentes com idade inferior a 24 anos foram a única categoria de idade estatisticamente significativa para a análise da ansiedade. Nesse sentido, observa-se que o coeficiente da dummy é estatisticamente siginificante e positivo, indicando que ter idade inferior a 24 anos faz com que a ansiedade seja maior, em comparação com os demais grupos.

A segunda variável que se apresentou estatisticamente significativa foi a variável gênero. Resultados de estudos anteriores apontam que os escores de ansiedade variam de acordo com o gênero (Andrade, Gorenstein, Viera Filho, Tung, \& Artes, 2001; Borine, 2011; Fioravanti, 2006). Nesse sentido, esta análise mostra que, para os mestrandos e doutorandos de Contabilidade, esse comportamento também foi observado. 
O coeficiente da variável dummy de gênero é negativo, sinalizando que os discentes do gênero masculino são menos ansiosos em comparação aos discentes do gênero feminino. Esse resultado é aderente com o encontrado no teste de média. Segundo Lewinsohn, Gotlib, Lewinsohn, Seeley e Allen (1998), essa diferença é atribuída a fatores genéticos e biológicos existentes entre os gêneros, e a diferenças que se originam nas experiências vivenciadas na contrução social de homens e mulheres.

A variável tipo de pós-graduação (se mestrado ou doutorado) também demonstrou ser relevante para a análise da ansiedade, tendo indicado que os mestrandos são mais ansiosos do que os doutorandos. Esse resultado difere do encontrado por Simon e Thomas (1983), que não encontraram diferenças estatisticamente significativas ao analisar sua amostra por tipo de curso e instituição. A variável desempenho apresentou-se estatisticamente significativa, no entanto o sinal encontrado foi positivo, ou seja, isso indica que há uma maior ansiedade naqueles que possuem pontuação mais alta. Pesquisas futuras são necessárias para verificar esse achado.

A variável 'suporte’também apresentou-se estatisticamente significativa. No entanto e surpreendentemente, o sinal dos coeficientes apresentou-se positivo. Ou seja, receber apoio psicológico/psiquiátrico e/ou receber bolsa acadêmica para cursar o mestrado e doutorado contribuem para o aumento da ansiedade. Com relação à bolsa acadêmica, esse resultado é, em parte, considerável, isso porque, ao receber uma bolsa institucional ou não ter que arcar com a mensalidade, usualmente, o estudante é obrigado a manter determinado nível de desempenho, ou seja, a pressão por notas aumenta, o que influenciaria na sua ansiedade. De mesmo modo, as bolsas oferecidas por instituições de fomento, como as oferecidas pela Coordenação de Aperfeiçoamento de Pessoal de Nível Superior (CAPES), têm como contrapartida a obrigatoriedade do discente de exercer o estágio docente. Isso significa que, além da demanda da pós-graduação, o estudante deve exercer outras atividades, o que explicaria o aumento da ansiedade quando o discente recebe esse tipo de suporte.

Entretanto esperava-se que a procura do apoio psicológico/psiquiátrico impactasse negativamente na ansiedade, já que, segundo Clark e Beck (2012), há diversas terapias cognitivas e comportamentais que auxiliam no controle e diminuição da ansiedade. Talvez, esse resultado demonstre que os discentes que procuram esse tipo de apoio são os que já demonstram maiores escores de ansiedade, o que induziu ao sinal positivo da dummy. Assim sendo, esse resultado merece aprofundamento futuro para se confirmar essa hipótese.

Com relação ao efeito da motivação na ansiedade, observa-se que a variável 'motivação para prosseguir nos estudos' impacta negativa e significativamente a ansiedade. Ou seja, quanto mais motivado para continuar estudando menor a ansiedade experimentada pelo discente. Esse é um indício inicial de que a motivação, ainda que analisada sem se considerar as sete dimensões, impacta negativamente a ansiedade.

A autoeficácia também impacta estatística e significativamente a ansiedade. Dessa maneira, quanto maior a percepção de autoeficácia, menor será a ansiedade experimentada. Assim, em consonância com a proposição teórica de Bandura (1977), uma maior percepção de autoeficácia é capaz de reduzir os níveis de ansiedade. Dessa maneira, não rejeitou-se a hipótese $\mathbf{H}_{\mathbf{1}}$ : A autoeficácia impacta negativa e significativamente a ansiedade.

No entanto, das dimensões de motivações extrínsecas, somente a motivação extrínseca por controle mostrou-se estatisticamente significativa para a análise da ansiedade. Assim, rejeitou-se a hipótese $\mathbf{H}_{2}$, pois, ao contrário do hipotetizado, a motivação extrínseca por identificação e a motivação extrínseca por introjeção não são estatisticamente significativas para a análise da ansiedade. Entretanto, observa-se que, dentro da proposição do modelo hierárquico de Vallerand (1997), a motivação extrínseca por controle foi a que apresentou consequências mais negativas, sendo menos prejudicial apenas do que a desmotivação, e essa motivação mostrou-se significativa para a análise da ansiedade. Isto é, quanto maior a motivação por controle externo, maior a ansiedade experimentada.

Nesse sentido, a regressão também indicou que a desmotivação é positiva e estatisticamente significativa para a análise, não se rejeitando a hipótese $\mathbf{H}_{3}$ : A desmotivação impacta positiva e significativamente a ansiedade. Ou seja, conforme a proposição de Vallerand (1997), a ansiedade sofre influência positiva e significativa da desmotivação, confirmando-se, dessa maneira, o teorizado no início deste estudo de que também para os mestrandos e doutorandos em Contabilidade, certos tipos de motivação podem apresentar consequência negativas para o estudante. Para o contexto estudado, isso significa dizer que, ao se sentir desmotivado, ou ao cursar a pósgraduação para obter recompensas externas ou evitar restrições (por exemplo: punição dos pais, julgamentos e frustrações), o estudante experimentará uma elevação na sua ansiedade.

Por fim, com relação às motivações intrínsecas, nenhuma das dimensões foram estatisticamente significativas para a análise da ansiedade, portanto, rejeitou-se a hipótese $\mathbf{H}_{4}$. Dessa forma, não foi possível concluir que a ansiedade sofre influência positiva e significativa das dimensões motivacionais, que deveriam reduzir a ansiedade, ou seja, da Motivação Intrínseca para saber, da Motivação Intrínseca para realização ou da Motivação intrínseca para vivenciar estímulos. 


\section{CONSIDERAÇÕES FINAIS}

Considerando o objetivo desse trabalho, de analisar o efeito que a motivação, a autoeficácia e variáveis sociodemográficas exercem sobre a ansiedade dos mestrandos e doutorandos em Contabilidade e partindo de uma amostra composta por 246 mestrandos e 76 doutorados dos programas de pós-graduação (mestrado e doutorado) em Contabilidade do Brasil, após a coleta, o estudo e a análise dos dados, entende-se cumprido o proposto, passando-se às considerações.

Concluiu-se que a ansiedade é negativa e significativamente impactada pela autoeficácia, e positiva e significativamente impactada pela motivação extrínseca por controle externo e pela desmotivação. Além disso, concluiu-se que são preditores significativos para a análise da ansiedade o nível de motivação para prosseguir nos estudos, ter idade abaixo de 24 anos, o tipo de pós-graduação (se mestrado ou doutorado), receber apoio psiquiátrico/psicológico, receber bolsa acadêmica, o desempenho e o gênero.

Diante desses achados, nota-se que a principal contribuição marginal do estudo consiste em apresentar resultados que embasem, numericamente, a discussão e avaliação dessas variáveis psicológicas para o contexto educacional do mestrado e doutorado em Contabilidade, pois, embora muitas pesquisas tenham sido feitas com essa variável, poucas focaram especificamente na pós-graduação dessa relevante área de conhecimento.

Entende-se, por isso, que as instituições de ensino precisam fomentar e aperfeiçoar as políticas de assistência e aconselhamento psicológico, que incluam os alunos de pós-graduação. As falhas nesse sistema de suporte são percebidas, por exemplo, quando se constata que estudantes que buscaram apoio psicológico tiveram sua ansiedade aumentada, conforme se apurou nesta pesquisa. Desse modo, além de trabalhar grupos de suporte para o compartilhamento de experiências, que propiciem a aproximação dos alunos e dos docentes (Oliveira, 2019; Tyssen \& Vaglum, 2002), as IES precisam criam ambientes acolhedores, que facilitem a integração dos estudantes à comunidade acadêmica, de modo a tornar sua experiência enriquecedora, tanto nos aspectos acadêmico e profissional, quanto no âmbito pessoal. Isso, certamente, possibilitará uma formação plena aos estudantes e eficaz do ponto de vista dos gastos públicos.

O primeiro passo que as IES podem dar, de modo a melhorar a situação encontrada nesta pesquisa, é a orientação. Os alunos de pós-graduação devem/precisam receber informações sobre reações emocionais e comportamentais comuns, que ocorrem na pós-graduação e em locais dentro e fora do campus, o que lhes permitirá aprimorar sua capacidade de autoavaliação e, se for o caso, buscar, de forma mais célere, tratamentos adequados. Ainda mais, seria recomendável que as IES, periodicamente, aproveitassem esse momento e espaço para encorajar e motivar os estudantes. Ao constatar que o sentimento de autoeficácia pode contribuir para minorar a ansiedade, a pesquisa revelou um importante mecanismo de controle, conforme já era advogado por Bandura (1977). Assim, considerando que a percepção de autoeficácia pode ser majorada por incentivos externos (Bandura, 1977), os professores e, em especial, os orientadores, podem, ainda que por meio de incentivos orais, contribuir para modular a ansiedade dos pós-graduandos.

Para além disso, toda a comunidade acadêmica (gestores, professores, técnicos e estudantes) deve estar atenta aos sinais de alerta de suicídio e de seus fatores de riscos (Tyssen \& Vaglum, 2002). Em todo o mundo, o suicídio é uma das três principais causas de morte entre pessoas na faixa etária economicamente mais produtiva (15-44 anos) (Patton et al., 2009, Garcia-Williams et al., 2014), notando-se, ao considerar a idade média dos respondentes ( 38 anos), que os estudantes de pós-graduação de Contabilidade integram um grupo no qual os altos níveis de sofrimento mental fomentam o pensamento suicida. Dessa forma, promover cursos, debates, treinamentos e outras formas de capacitar a comunidade para identificar o estudante em sofrimento mental não só contribui para a humanização do ambiente acadêmico e, certamente, para a realização pessoal dos estudantes e para a sua felicidade, mas também para a prevenção ao suicídio.

Por último, considera-se que pesquisas futuras possam ser desenvolvidas em relação a estratégias de enfrentamento à ansiedade de modo a minorá-la e promover maior bem-estar aos estudantes. Nessas pesquisas, abordagens que tratem de mecanismos de fomento e ampliação de sentimentos relacionados à autoeficácia poderiam ser propostos e testados de modo a medir sua efetividade, especialmente abordagens que desse especial atenção às mulheres. Outra possibilidade seria investigar a relação da ansiedade com a depressão, que poderia estar acomentendo os estudantes com níveis maiores de ansiedade. 


\section{REFERENCIAS}

Andrade, L., Gorenstein, C., Viera Filho, A. H., Tung, T. C., \& Artes, R. (2001). Psychometric properties of the Portuguese version of the State-Trait Anxiety Inventory applied to college students: factor analysis and relation to the Beck Depression Inventory. Brazilian Journal of Medical and Biological Research, 34(3), 367-374.

Andrade, A. D. S., Tiraboschi, G. A., Antunes, N. A., Viana, P. V. B. A., Zanoto, P. A., \& Curilla, R. T. (2016). Vivências acadêmicas e sofrimento psíquico de estudantes de Psicologia. Psicologia: ciência e profissão, 36(4), 831-846.

Araujo, I. R. de. (2015). A motivação de licenciandos em música sob a perspectiva da teoria da autodeterminação. Universidade Federal do Rio Grande do Norte.

Bandura, A. (1977). Self-efficacy: Toward a unifying theory of behavioral change. Psychological Review, 84(2), 191-215. DOI: https://doi.org/10.1037/0033-295x.84.2.191

Betz, N. E. (1978). Prevalence, distribution, and correlates of math anxiety in college students. Journal of Counseling Psychology, 25(5), 441-448. DOI: https://doi.org/10.1037/0022-0167.25.5.441

Biaggio, A. M. B., Natalício, L., \& Spielberger, C. D. (1977). Desenvolvimento da forma experimental em português do Inventário de Ansiedade Traço-Estado (IDATE) de Spielberger. Arquivos Brasileiros de Psicologia Aplicada, 29(3), 31-44.

Borine, M. S. (2011). Ansiedade, neuroticismo e suporte familiar: Evidência de validade do Inventário de Ansiedade Traço-Estado (IDATE). Universidade São Francisco. Retrieved from http://dsv.usf.edu.br/galeria/ getImage/427/606054467274901.pdf

Bueno, M. (2002). As Teorias de motivação humana e a sua contribuição para a empresa humanizada: um tributo a Abraham Maslow. Revista do Centro de Ensino Superior de Catalão - CESUC, 6, 1-25.

Bzuneck, J. A. (2001). As crenças de auto-eficácia e o seu papel na motivação do aluno. In E. Vozes (Ed.), A motivação do aluno: contribuições da psicologia contemporânea. (Vol. 1o, pp. 166-133). Petrópolis. Retrieved from http://www.uky.edu/ eushe2/Pajares/Bzuneck2.pdf

Castillo, A., Recondo, R., Asbahr, F. R., \& Manfro, G. (2000). Transtornos de ansiedade. Revista Brasileira de Psiquiatria (Supl II), 22(m), 20-25. DOI: https://doi.org/10.1590/S1516-44462000000600006

Clark, D. A., \& Beck, A. T. (2012). Terapia cognitiva para os transtornos de ansiedade. Porto Alegre: Artmed.

Conselho de Educação Superior (1965). Parecer n. 977/65. Definição dos cursos de pós-graduação necessidade da pós-graduação.

Conselho Federal de Contabilidade (2003). Legislaçaõ da profissão contábil (1st ed.). Brasília: Conselho Federal de Contabilidade. Retrieved from https://cfc.org.br/wp-content/uploads/2015/12/legis_profcontabil.pdf

Evans, T. M., Bira, L., Gastelum, J. B., Weiss, L. T., \& Vanderford, N. L. (2018). Evidence for a mental health crisis in graduate education. Nature Biotechnology, 36(3), 282-284. DOI: https://doi.org/10.1038/nbt.4089

Fairchild, A. J., Horst, S. J., Finney, S. J., \& Barron, K. E. (2005). Evaluating existing and new validity evidence for the Academic Motivation Scale. Contemporary Educational Psychology, 30(3), 331-358. DOI: https:// doi.org/10.1016/j.cedpsych.2004.11.001

Fioravanti, A. C. M. (2006). Propriedades Psicométricas do Inventário de Ansiedade Traço-Estado. Pontifícia Universidade Católica do Rio de Janeiro.

Garcia-Williams, A. G., Moffitt, L., \& Kaslow, N. J. (2014). Mental health and suicidal behavior among graduate students. Academic psychiatry, 38(5), 554-560.

Horta, R. L., Horta, B. L., \& Horta, C. L. (2012). Uso de drogas e sofrimento psíquico numa universidade do Sul do Brasil. Psicologia em Revista, 18(2), 264-276.

Iqbal, S., Gupta, S., \& Venkatarao, E. (2018). Stress , anxiety \& depression among medical undergraduate students \& their socio-demographic correlates. Indian Journal of Medical Research, 141(3), 354-357.

Iudícibus, S. de, Martins, E., \& Carvalho, L. N. (2005). Contabilidade: Aspectos relevantes da epopeia de sua evolução. Revista Contabilidade \& Finanças, 38, 7-19.

Lei n. 9.394 (1996). Estabelece as diretrizes e bases da educação nacional. Brasília. 
Levecque, K., Anseel, F., Beuckelaer, A., Van der Heyden, J., \& Gisle, L. (2017). Work organization and mental health problems in PhD students. Research Policy, 46(4), 868-879. DOI: https://doi.org/10.1016/j. respol.2017.02.008

Lewinsohn, P. M., Gotlib, I. H., Lewinsohn, M., Seeley, J. R., \& Allen, N. B. (1998). Gender Differences in Anxiety Disorders and Anxiety Symptoms in Adolescents. Journal of Abnormal Psychology, 107(I), 109-117. DOI: https://doi.org/10.1037//0021-843X.107.1.109

Maslow, A. H. (1943). A theory of human motivation. Psychological Review, 50(4), 370-396. DOI: https://doi. org/10.1037/h0054346

Neiva, J. F. de O. (2010). Estabelecimento de metas e ansiedade traço na aquisição de habilidades motoras. Universidade de São Paulo.

Nogueira, C., \& Mesquita, A. P. (1992). Autoeficácia e ansiedade: aplicações na consulta psicológica. Jornal de Psicologia, 10(3), 16-22.

Oliveira, C. A. D. (2019). Ansiedade, depressão e estresse, uso de álcool e outras drogas e a satisfação de discentes de pós-graduação stricto sensu. Dissertação de Mestrado, Universidade Federal do Amapá, Macapá, AP, Brasil.

Patton, G. C., Coffey, C., Sawyer, S. M., Viner, R. M., Haller, D. M., Bose, K., ... \& Mathers, C. D. (2009). Global patterns of mortality in young people: a systematic analysis of population health data. The lancet, 374(9693), 881-892.

Reis, C. F., Miranda, G. J., \& Freitas, S. C. (2017). Ansiedade e desempenho acadêmico : Um estudo com alunos de Ciências Contábeis. Advances in Scientic and Applied Accouting, 10(3), 319-333.

Ryan, R. M., \& Deci, E. L. (2000). Intrinsic and extrinsic motivations: Classic definitions and new directions. Contemporary Educational Psychology, 25(1), 54-67. DOI: https://doi.org/10.1006/ceps.1999.1020

Schunk, D. H. (1991). Self-efficacy and Academic Achievement. Sociological Perspectives, $26(3$ \& 4), $207-231$. DOI: https://doi.org/10.1177/0731121416629993

Schunk, D. H., \& Pajares, F. (2001). The development of academic Self-Efficacy. In A. Wigfield \& J. S. Eccles (Eds.), Development of achievement motivation (7th ed.). San Diego: Academic Press.

Schwarzer, R., Bäßler, J., Kwiatek, P., Schröder, K., \& Zhang, J. X. (1997). The assessment of optimistic selfbeliefs: Comparison of the German, Spanish, and Chinese versions of the general self-efficacy scale. Applied Psychology, 46(1), 69-88. DOI: https://doi.org/10.1111/j.1464-0597.1997.tb01096.x

Serpa, A. L. de O. (2012). Autoeficácia, autoconceito e ansiedade em uma avaliação em larga escala e sua relação com o desempenho escolar. Universidade Federal de Juiz de Fora.

Simon, A., \& Thomas, A. (1983). Test data for the State-Trait Anxiety Inventory for British Further Education, Certificate of Education and B.Ed. students. Personality and Individual Differences, 4(2), 199-200. DOI: https://doi.org/10.1016/0191-8869(83)90020-X

Smith, R. E. (1989). Effects of coping skills training on generalized self-efficacy and locus of control. Journal of Personality and Social Psychology, 56(2), 228-233. DOI: https://doi.org/10.1037/0022-3514.56.2.228

Sobral, D. T. (2003). Motivação do aprendiz de medicina: Uso da Escala de Motivação Acadêmica. Psicologia: Teoria e Pesquisa, 19(1), 25-31. DOI: https://doi.org/10.1590/S0102-37722003000100005

Spielberger, C. D. (Ed.). (1966). Anxiety and Behavior. New York and London: Academic Press.

Spielberger, C. D. (1972). Anxiety: Current Trends in Theory and Research. Anxiety as an Emotional State, 250. DOI: https://doi.org/10.1017/CBO9781107415324.004

Spielberger, C. D., \& Gorsuch, R. L. (1966). Mediating processes in verbal conditioning. Report to National Institute of Mental Health.

Spielberger, C. D., Gorsuch, R. L., \& Lushene, R. E. (1970). Manual for the state-trait anxiety inventory.

Spielberger, C. D., Gorsuch, R. L., \& Lushene, R. E. (1979). Inventário de ansiedade traço-estado. Rio de Janeiro: CEPA.

Teixeira, J., Dias, A., \& Dell'Aglio, D. (2012). Propriedades Psicométricas da Escala de Autoeficácia Geral Percebida (EAGP). Psico, 43(7), 139-146. DOI: https://doi.org/10.1111/j.1746-1561.2005.00034.x 
Tyssen, R., \& Vaglum, P. (2002). Mental health problems among young doctors: an updated review of prospective studies. Harvard review of psychiatry, 10(3), 154-165.

Vallerand, R. J. (1997). Toward a hierarchical model of intrinsic and extrinsic motivation. Advances in Experimental Social Psychology, 29, 271-360. DOI: https://doi.org/10.1016/S0065-2601(08)60019-2

Vallerand, R. J., Pelletier, L. G., Blais, M. R., Briere, N. M., Senecal, C., \& Vallières, É. F. (1992). The academic motivation scale: A measure of intrinsic, extrinsic, and amotivation in education. Educational and Psychological Measurement, 52, 1003-1017. DOI: https://doi.org/10.1177/0013164492052004025

Viana, G. S. (2012). Atitude e motivação em relação ao desempenho acadêmico de alunos do curso de graduação em administração em disciplinas de estatística. Universidade de São Paulo.

Weiner, B. (1990). History of motivational research in education. Journal of Educational Psychology, 82(4), 616622. DOI: https://doi.org/10.1037/0022-0663.82.4.616

Weiner, I. B., \& Craighead, W. E. (Eds.). (2009). The Corsini Encyclopedia of Psychology (3rd ed.). John Wiley \& Sons Inc.

White, W. R. (1975). Motivation reconsidered: The concept of competence. In P. H. Mussem, J. J. Conger, \& J. Kagan (Orgs.) (Eds.), Basic and contemporary issues in developmental psychology (pp. 230-266). Harper \& Row.

Witter, G. P. (1984). Aprendizagem e motivação. In G. P. Witter \& J. F. P. Lomanaco (Eds.), Psicologia da Aprendizagem (pp. 37-57). São Paulo: EPU.

Wooldridge, J. M. (2010). Econometric analysis of cross section and panel data. MIT press.

\section{Como citar este artigo}

Coelho, W. E.; \& Nascimento, E. M. (2020). A ansiedade dos mestrandos e doutorandos em contabilidade. Revista de Contabilidade e Organizações, 14:e172020. DOI: http://dx.doi.org/10.11606/issn.1982-6486. rco.2020.172020 